\title{
A New Image Fusion Method based on Integration of Wavelet and Fast Discrete Curvelet Transform
}

\author{
Anjali A. Pure \\ ECE dept. \\ TIEIT, Bhopal, India.
}

\author{
Neelesh Gupta \\ HOD, ECE dept. \\ TIEIT, Bhopal, India.
}

\author{
Meha Shrivastava \\ Asst. Professor, ECE dept. \\ TIEIT, Bhopal, India.
}

\begin{abstract}
Image fusion is one of the most useful term related to digital image processing, computer vision and medical imaging. The objective of image fusion is to extract the useful information from several images into a single image. Recently, more research has been done on wavelet based image fusion methods for medical application. Wavelet transform is useful for objects with point singularities and analyses the feature of images in detailed, but it does not provide information about edges clearly. While curvelet transform is more useful for the analysis of images having curved shape edges. So, in this paper, a new image fusion method is proposed based on the integration of wavelet and fast discrete curvelet transform, which describe the curved shapes of images and analyses feature of images better. This paper uses MRI and CT images for fusion which contains complementary information helpful for diagnosis of disease. The fusion results obtained from proposed method are analyzed and compared visually and statistically with different types of wavelets used in image fusion. The results of proposed method are efficient and improve the Entropy, PSNR, Mean, STD and MSE. The proposed method can be helpful for better medical diagnosis.
\end{abstract}

\section{Keywords}

Image fusion, MRI Image, CT image, Discrete Wavelet Transform (DWT), Fast Discrete Curvelet Transform (FDCT).

\section{INTRODUCTION}

Image fusion is very important technique used to extract the useful information from several images into a single image. Till date, many image fusion methods have been developed such as, the Brovey, the HIS and PCA methods. These methods operate under spatial domain $\&$ have proved to be successful in computer vision, robotics, satellite and medical image fusion applications [1]. Now-a-days, Medical image fusion has become a new promising research field. For medical diagnosis, MRI (Magnetic resonance image) and CT (Computed tomography) images are very important. MRI image provides better information about soft tissue and CT image provides detail information about dense structure such as bones. These two images provide complementary information. The main purpose of medical image fusion is to obtain a high resolution image with as much details as possible for the sake of diagnosis. So if these two images of the same organ are fused then the fused image contains as much information as possible for diagnosis of that organ [2]. Researchers have made lot of work on the fusion of MRI \&CT images using wavelet transform. Jean Morlet in 1982 introduced the idea of the wavelet transform. Three types of wavelets used in the image fusion are Orthogonal, Biorthogonal and A'trous (Non-orthogonal). The image fusion method based on wavelet transform has good spatial \& spectral quality but has limited directivity to deal with the images having curved shapes $[3,8]$. So, it is necessary to develop an advanced image fusion method which provides detail information about curved shapes. E. J. Candes and D. L. Donoho put forward Fast Discrete Curvelet Transform in 2005 [6]. The curvelet transform is a multiscale directional transform that gives optimal non-adaptive sparse representation of objects with edges and well-suited for multiscale edge enhancement [7].

Literature survey of image fusion methods for medical applications reveals that most of the image fusion methods are carried out using traditional wavelet transform and curvelet transform separately $[2,3]$. Until now more research work is done on comparison of wavelet and curvelet transform. Recently, image fusion with fast discrete curvelet transform is used to improve fusion results. The objective of this paper is to integrate discrete wavelet transform and fast discrete curvelet transform which describe the curved shapes of object and analyses feature of images better. The results of proposed method are compared with three types of wavelets used in image fusion at last.

The rest of the paper is organized as: Section 2 presents the proposed image fusion method. Quantative analysis is given in section 3. Section 4 gives the experimental results. Finally, conclusion is given in section 5 .

\section{PROPOSED IMAGE FUSION METHOD}

Wavelet transform is useful for objects with point singularities and analyses the feature of images in detailed, but it does not provide information about edges clearly. While curvelet transform is more useful for the analysis of images having curved shape edges. So, in this paper, a new image fusion method is proposed based on the integration of discrete wavelet transform and fast discrete curvelet transform, which describe the curved shapes of images and analyses feature of images better. In medical image fusion, edges play a very important role. This paper uses MRI and CT images for fusion. These two images contain complementary information. Images can be fused to get as much information as possible from input image. 


\subsection{Block Diagram}

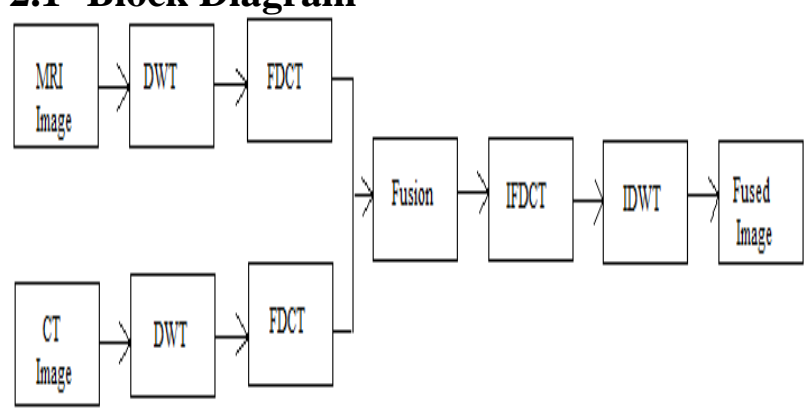

Figure 1: Block diagram of Proposed Image Fusion Method

Figure 1 shows block diagram of proposed image fusion method. Firstly, 2D-discrete wavelet transform is applied on both the input images. It decomposes input images into four subbands. One low frequency approximate component and three high frequency detail components will be acquired in each Subband which preserves better information content from input images. Fast discrete curvelet transform using frequency wrapping is then applied to low frequency approximate component and high frequency detail components to obtain curvelet coefficients by calculating image orientation from different angles. Then these coefficients are fused using maximum selection rule which selects maximum valued pixel. The fused coefficients are obtained after fusion process. The fused image is obtained by applying inverse fast discrete curvelet transform on fused coefficients. The final fused image is obtained by applying inverse discrete wavelet transform on fused image. Comparative analysis is done on fused images obtained from both integration of discrete wavelet and fast discrete curvelet transform and different types of wavelets used in image fusion. The Performance can be evaluated by using Entropy, RMSE, PSNR, Mean and STD.

\subsection{Proposed Image Fusion Algorithm}

The proposed image fusion algorithm is implemented for fusion of medical images. In medical imaging, MRI and CT images are of main concern for diagnosis of brain, chest and spines related diseases. MRI image provides better information about soft tissue and CT image provides detail information about dense structure such as bones. These two images provide complementary information. Here fusion of these two images plays a very important role which combines both CT and MRI images into a single fused image which contains accurate information about bones as well as soft tissue details. Thus, the fusion of these two images of same organ provides very useful information about that organ which helps physicians for better diagnosis.

The steps involved in proposed algorithm can be summarized as follows:

1. Read the MRI as image1 [M1, N1] and CT as image2 [M2, N2] which is to be fused.

2. Resample and register both these images, so that wavelet coefficients of similar component will stay in the same magnitude.

3. Apply 2D-discrete wavelet transform to these images which decompose it into four sub-bands (LL, LH, HL and HH). These four sub-bands are sensitive to low frequency approximate component and three high frequency detailed components.
4. The wavelet coefficients from both the input images are obtained which gives high spatial resolution and high spectral quality contents from input images.

5. Further Fast discrete curvelet transform using frequency wrapping is applied to obtain curvelet coefficients.

6. The steps for FDCT using frequency wrapping algorithm are explained as follows-

- Apply 2D FFT transform to both input images and obtain fourier samples of both images as $I 1[\mathrm{n} 1, \mathrm{n} 2]$ and $\mathrm{I} 2[\mathrm{n} 1, \mathrm{n} 2]$ where $-\mathrm{n} / 2 \leq \mathrm{n} 1, \mathrm{n} 2<\mathrm{n} / 2$. The obtained frequency samples of both images are periodized.

- For each scale $\mathrm{j}$ and angle a the periodization of widowed data is done which form the product for input image $\mathrm{I} 1[\mathrm{n} 1, \mathrm{n} 2]$ as

$$
\mathrm{K}_{1}[\mathrm{n} 1, \mathrm{n} 2]=\mathrm{U}_{\mathrm{j}, \mathrm{a}}[\mathrm{n} 1, \mathrm{n} 2] \mathrm{I} 1[\mathrm{n} 1, \mathrm{n} 2]
$$

And input image $\mathrm{I} 2[\mathrm{n} 1, \mathrm{n} 2]$ as

$$
\mathrm{K}_{2}[\mathrm{n} 1, \mathrm{n} 2]=\mathrm{U}_{\mathrm{j}, \mathrm{a}}[\mathrm{n} 1, \mathrm{n} 2] \mathrm{I} 2[\mathrm{n} 1, \mathrm{n} 2]
$$

- $\quad$ The obtained windowed data, wrappedK $\mathrm{K}_{1}[\mathrm{n} 1, \mathrm{n} 2]$ and $\mathrm{K}_{2}[\mathrm{n} 1, \mathrm{n} 2]$ around the origin to restrict the rectangular window length L1,j and L2,j near the origin. The product obtained is

$$
\begin{aligned}
& \mathrm{I}_{\mathrm{j}, \mathrm{a}}[\mathrm{n} 1, \mathrm{n} 2]=\mathrm{W}\left(\mathrm{U}_{\mathrm{j}, \mathrm{a}} \mathrm{I} 1\right)[\mathrm{n} 1, \mathrm{n} 2] \\
& \mathrm{I} 2_{\mathrm{j}, \mathrm{a}}[\mathrm{n} 1, \mathrm{n} 2]=\mathrm{W}\left(\mathrm{U}_{\mathrm{j}, \mathrm{a}} \mathrm{I} 2\right)[\mathrm{n} 1, \mathrm{n} 2]
\end{aligned}
$$

Where the range for $\mathrm{n} 1, \mathrm{n} 2$ is $0 \leq \mathrm{n} 1<\mathrm{L} 1, \mathrm{j}, 0 \leq \mathrm{n} 2<\mathrm{L} 2$, $\mathrm{j}$ (for- $\pi / 4 \leq \theta<\pi / 4$ ).

Thus, the wrapping transformation is nothing but a simple reindexing of the data.

- $\quad$ Apply the inverse 2D FFT to each $\mathrm{I} 1_{\mathrm{j}, \mathrm{a}}$ and $\mathrm{I} 2_{\mathrm{j}, \mathrm{a}}$.

- The curvelet coefficients $I 1_{\mathrm{j}, \mathrm{a}}$ and $\mathrm{I} 2_{\mathrm{j}, \mathrm{a}}$ of both the input images are obtained which contains high directionality.

- These coefficients are fused using maximum selection rule.

- For Maximum selection rule, fusion is done by taking the maximum valued pixels from I1 $[\mathrm{n} 1, \mathrm{n} 2]$ andI2[n1,n2] both sub images of input images.

$$
\mathrm{I}_{\max }=\max (\mathrm{I} 1[\mathrm{n} 1, \mathrm{n} 2], \mathrm{I} 2[\mathrm{n} 1, \mathrm{n} 2])
$$

Fused coefficients are obtained.

7. The fused image is obtained by applying inverse fast discrete curvelet transform on fused coefficients.

8. The final fused image is reconstructed by applying inverse discrete wavelet transform to fused image.

9. The final fused image can be represented by following equation:

$$
\mathrm{I}[\mathrm{n} 1, \mathrm{n} 2]=\mathrm{W}^{1}(\Psi(\mathrm{W}(\mathrm{I} 1[\mathrm{n} 1, \mathrm{n} 2])), \mathrm{W}(\mathrm{I} 2[\mathrm{n} 1, \mathrm{n} 2)))
$$

10. Comparative analysis is done by using 5 quality metrics parameters as Entropy, RMSE, PSNR, Mean and STD.

\section{QUANTITATIVE ANALYSIS}

Quantitative analysis is carried out for comparison of different image fusion methods based on discrete wavelet transform and fast discrete curvelet transform. In this paper, 5 different parameters are used as follows:

\subsection{Entropy}

The entropy of an image is a statistical measure of randomness that can be used to characterize the texture of the input image. It represents richness of information content in an image. The value of entropy should be large for better information in an image. 


\subsection{PSNR}

The PSNR (Peak Signal-to-Noise Ratio) is the most commonly used as quality of reconstruction of fused image. It represents the peak signal to noise ratio, so the value of PSNR must be high for less noise in an image. It is defined as,

$$
\mathrm{PSNR}=10 \log _{10}\left(255^{2} / \mathrm{MSE}\right)
$$

Where, 255 is the maximum pixel value of the image when the pixels are represented using 8-bit per sample.

\subsection{RMSE}

The RMSE represents the root mean square error which describes the difference in the pixel values between the corresponding pixels of the two images. The value of RMSE must be small for better fused image. For two MxN images $\mathrm{S}$ and $\mathrm{F}$, where image $\mathrm{S}$ is considered a source image and $\mathrm{I}$ is the fused image.

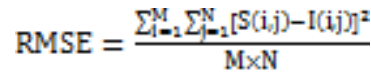

\subsection{Mean}

The mean represents the average of pixel values of an image So, the value of Mean should be high for better contrast in an fused image.

\subsection{Standard deviation (STD)}

Standard deviation is the square root of variance, reflects the spread in the data. It represents the deviation of pixel values from mean, so the value of STD should be high for better contrast of an image.

\section{EXPERIMENTAL RESULTS}

The experimental results of proposed image fusion method for fusion of medical images are shown in figures. Figure 2 represent MRI image of brain which provides better information on soft tissues. Figure 3 represent CT image of brain of same person which provide the best information of dense structures like bones but misses soft tissue information. Figure 4 shows result of orthogonal wavelet based fusion method. The fused image contains information of both images but aliasing effect is more. Figure 5 shows result of Biorthogonal wavelet based fusion method. The fused image contains more information on soft tissue. Figure 6 is the result of A'trous (non-orthogonal) wavelet based fusion method. The fused image contains less information of both soft and denser tissue. Figure 7 is the result of proposed image fusion method which shows detailed information of soft and denser tissues of the brain. Also comparative analysis of image fusion methods by DWT and proposed method is shown in Table 1 . The 5 different quality metrics parameters are calculated for fused images obtained for image fusion methods by DWT and proposed method. Visually, simulation results obtained for proposed image fusion method have higher values of Entropy and PSNR and lower value of RMSE. So the fused image obtained for proposed image fusion method have more information content in fused image, better PSNR and low RMSE value. Thus, the proposed image fusion method proved to be very efficient for medical imaging applications.

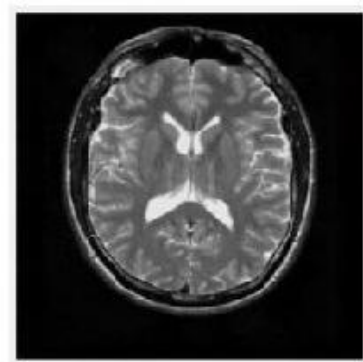

Figure 2: MRI image

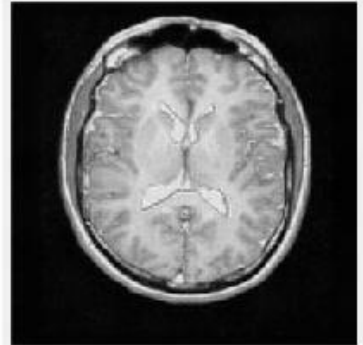

Figure 4: Orthogonal fused image

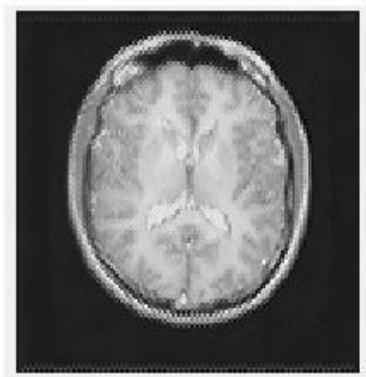

Figure 6: A'trous fused image

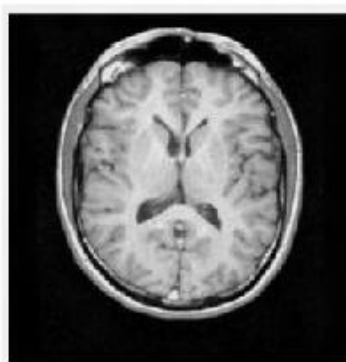

Figure 3: CT image

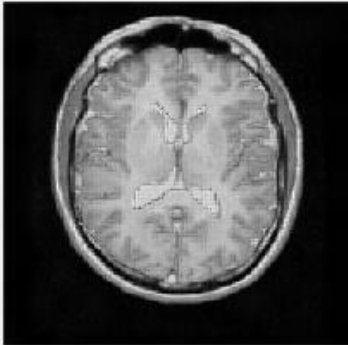

Figure 5: Biorthogonal fused image

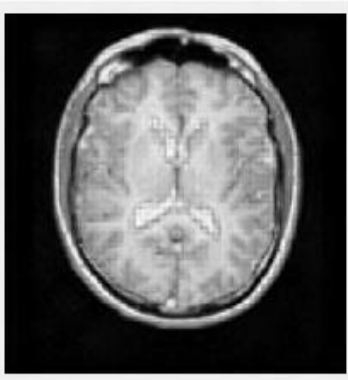

Figure 7: Wavelet \& FDCT fused image

\begin{tabular}{|c|c|c|c|c|}
\hline Methods & $\begin{array}{l}\text { Orthog } \\
\text { onal }\end{array}$ & $\begin{array}{l}\text { Biortho- } \\
\text { gonal }\end{array}$ & A'trous & $\begin{array}{l}\text { Wavelet } \\
\& \\
\text { FDCT }\end{array}$ \\
\hline Entropy & 1.30572 & 0.96129 & 1.98314 & 6.24702 \\
\hline PSNR & 30.8992 & 30.8751 & 32.5359 & 43.4797 \\
\hline MSE & 52.8643 & 53.1579 & 36.2651 & 2.9182 \\
\hline Mean & 79.6636 & 79.7596 & 37.299 & 79.2667 \\
\hline $\begin{array}{l}\text { Standard } \\
\text { deviation }\end{array}$ & 85.7467 & 85.9938 & 42.2116 & 84.6976 \\
\hline
\end{tabular}

Table 1. Quantitative analysis of fusion methods

\section{CONCLUSION}

The proposed image fusion method based on integration of discrete wavelet transform and fast discrete curvelet transform works efficiently for fusion of medical imaging applications. 
Quantitative analysis shows better results for proposed method with higher values of Entropy and PSNR and minimum value of RMSE than different types of wavelet used in image fusion method. Visually, the results of proposed image fusion method preserve both high spatial resolution and high spectral quality contents of an image with good directionality and best suited for medical applications. The obtained results can be used by researchers for further research work on image fusion.

\section{REFERENCES}

[1] Shih-Gu Huang, Wavelet for Image Fusion.

[2] Smt. G. Mamatha, L. Gayatri, 'AN IMAGE FUSION USING WAVELET AND CURVELET TRANSFORMS', Global Journal of Advanced EngineeringTechnologies, Vol1,Issue-2,2012,ISSN:22776370 .

[3] A. Soma Sekhar, Dr. M. N. GiriPrasad, 'A Novel Approach of Image Fusion on MR and CT Images Using Wavelet Transforms, IEEE 2011.

[4] S.Bharath and E.S. Karthik Kumar, Implementation Of Image Fusion Algorithm Using 2gcurvelet Transforms, ISBN 978-1-4675-2248-9@2012.

[5] Bin Yang and Shutao Li, Multifocus Image Fusion and Restoration with Sparse Representation, IEEE 2010.

[6] Emmanuel Cand'es, Laurent Demanet, David Donoho and Lexing Ying; Fast Discrete Curvelet Transforms,
Applied and Computational Mathematics, Caltech Pasadena 2006

[7] Jianwei Ma and Gerlind Plonka, The Curvelet Transform, IEEE SIGNAL PROCESSING MAGAZINE[118] MARCH 2010.

[8] Gang Hong, Yun Zhang, 'The effect of different types of wavelets on image fusion.

[9] Deepa M, Wavelet and Curvelet Based Thresholding Techniques for Image Denoising, IJARCSEE voll Issue2012, ISSN: 227-9043.

[10] E. J. Candes, D.L.Donoho Curvelets: A Surprisingly Effective Nonadaptive Representation for Objects with Edges.

[11] M. Sifuzzaman M.R. Islam, M. Z. Ali, Application Of Wavelet and its Advantages Compared to Fourier Transform, Journal of Physical Science,Vol.13,2009,121-134,ISSN:0972-8791.

[12] Y. Kiran Kumar, Comparison of Fusion Techniques Applied to preclinical images: Fast Discrete Curvelet Transform using Wrapping Technique \& Wavelet Transform, JATIT2009.

[13] Pao-Yen Lin, An introduction wavelet transforms

[14] Myungjin Choi, Rae Young Kim, Moon-GyuKim, 'The curvelet transform for image fusion.

[15] Rafael C. Gonzalez and Richard E. Woods, Digital image Processing, Third Edition. 